\title{
Strong Law of Large Numbers for a 2-Dimensional Array of Pairwise Negatively Dependent Random Variables
}

\author{
Karn Surakamhaeng $^{1}$, Nattakarn Chaidee ${ }^{1,2}$, Kritsana Neammanee ${ }^{1}$ \\ ${ }^{1}$ Department of Mathematics and Computer Science, Chulalongkorn University, Bangkok, Thailand \\ ${ }^{2}$ Centre of Excellence in Mathematics, CHE, Bangkok, Thailand \\ Email: nattakarn.c@chula.ac.th
}

Received October 30, 2012; revised November 30, 2012; accepted December 14, 2012

\begin{abstract}
In this paper, we obtain the strong law of large numbers for a 2-dimensional array of pairwise negatively dependent random variables which are not required to be identically distributed. We found the sufficient conditions of strong law of large numbers for the difference of random variables which independent and identically distributed conditions are regarded. In this study, we consider the limit as $m \times n \rightarrow \infty$ which is stronger than the limit as $m, n \rightarrow \infty$ when $m, n$ are natural numbers.
\end{abstract}

Keywords: Strong Law of Large Numbers; Negatively Dependent; 2-Dimensional Array of Random Variables

\section{Introduction and Main Results}

Let $\left(X_{i}\right)_{i \in N}$ be a sequence of random variables. We say that $\left(X_{i}\right)_{i \in N}$ satisfies the strong law of large numbers (SLLN) if there exist sequences of real numbers $\left(a_{n}\right)_{n \in N}$ and $\left(b_{n}\right)_{n \in N}$ such that $\frac{S_{n}-a_{n}}{b_{n}} \stackrel{\text { a.s. }}{\longrightarrow} 0$ as $n \rightarrow \infty$. where $S_{n}=\sum_{i=1}^{n} X_{i}$ and the abbreviation a.s. stands for almost surely.

To study the strong law of large numbers, there is a simple question come in mind. When does the sequence $\left(X_{i}\right)_{i \in N}$ satisfy the SLLN? Many conditions of the sequence $\left(X_{i}\right)_{i \in N}$ have been found for this question. The SLLN are investigated extensively in the literature especially to the case of a sequence of independent random variables (see for examples in [1-3]). After concepts of dependence was introduced, it is interesting to study the SLLN with condition of dependence.

A sequence $\left(X_{i}\right)_{i \in N}$ of random variables is said to be pairwise positively dependent (pairwise PD) if for any $a, b \in R$ and $i \neq j$,

$$
P\left(X_{i}>a, X_{j}>b\right) \geq P\left(X_{i}>a\right) P\left(X_{j}>b\right)
$$

and it is said to be pairwise negatively dependent (pairwise ND) if for any $a, b \in R$ and $i \neq j$,

$$
P\left(X_{i}>a, X_{j}>b\right) \leq P\left(X_{i}>a\right) P\left(X_{j}>b\right) .
$$

Theorem 1.1-1.5 are examples of SLLN for a sequence of pairwise $\mathrm{PD}$ and pairwise ND random variables.
Theorem 1.1. (Birkel, [4]) Let $\left(X_{i}\right)_{i \in N}$ be a sequence of pairwise PD random variables with finite variances. Assume

1) $\sup _{i \in N} E\left(\left|X_{i}-E\left(X_{i}\right)\right|\right)<\infty$,

2) $\sum_{i=1}^{n} \frac{\operatorname{Cov}\left(X_{i}, S_{i}\right)}{i^{2}}<\infty$.

Then $\frac{S_{n}-E\left(S_{n}\right)}{n} \stackrel{\text { a.s. }}{\longrightarrow} 0$ as $n \rightarrow \infty$.

Theorem 1.2. (Azarnoosh, [5]) Let $\left(X_{i}\right)_{i \in N}$ be a sequence of pairwise ND random variables with finite variances. Assume

1) $\sup _{i \in N} E\left(\left|X_{i}\right|\right)<\infty$,

2) $\sum_{i=1}^{n} \frac{\operatorname{Var}\left(X_{i}\right)}{i^{2}}<\infty$.

Then $\frac{S_{n}-E\left(S_{n}\right)}{n} \stackrel{\text { a.s. }}{\longrightarrow} 0$ as $n \rightarrow \infty$.

Theorem 1.3. (Nili Sani, Azarnoosh and Bozorgnia, [6]) Let $\left(a_{n}\right)_{n \in N}$ be a positive and increasing sequence such that $a_{n} \rightarrow \infty$ as $n \rightarrow \infty$.

Let $\left(X_{i}\right)_{i \in N}^{n}$ be a sequence of pairwise ND random variables with finite variances such that
1) $\sup _{n \in N}\left(\sum_{i=1}^{n} \frac{E\left(\left|X_{i}-E\left(X_{i}\right)\right|\right)}{a_{n}}\right)<\infty$,
2) $\sum_{i=1}^{\infty} \frac{\operatorname{Var}\left(X_{i}\right)}{a_{i}^{2}}<\infty$. 
Then $\frac{S_{n}-E\left(S_{n}\right)}{a_{n}} \stackrel{\text { a.s. }}{\longrightarrow} 0$ as $n \rightarrow \infty$.

In this work, we study the SLLN for a 2-dimensional array of pairwise ND random variables. We say that $\left(X_{i, j}\right)_{i, j \in N}$ satisfies the SLLN if there exist double sequences of real numbers $\left(a_{m, n}\right)_{m, n \in N}$ and $\left(b_{m, n}\right)_{m, n \in N}$ such that $\frac{S_{m, n}-a_{m, n}}{b_{m, n}} \stackrel{\text { a.s. }}{\longrightarrow} 0$ as $m, n \rightarrow \infty$ where $S_{m, n}=\sum_{i=1}^{m} \sum_{j=1}^{n} X_{i, j}$.

In 1998, Kim, Beak and Seo investigated SLLN for a 2-dimensional array of pairwise PD random variables and it was generalized to a case of weighted sum of 2-dimensional array of pairwise PD random variables by Kim, Baek and Han in one year later. The followings are their results.

A double sequence $\left(X_{i, j}\right)_{i, j \in N}$ is said to be pairwise positively dependent (pairwise PD) if for any $a, b \in R$ and $(i, j) \neq(k, l)$,

$$
P\left(X_{i, j}>a, X_{k, l}>b\right) \geq P\left(X_{i, j}>a\right) P\left(X_{k, l}>b\right)
$$

Theorem 1.4. (Kim, Beak and Seo, [7]) Let $\left(X_{i, j}\right)_{i, j \in N}$ be a 2-dimensional array of pairwise PD random variables with finite variances. Assume

1) $\sup _{i, j \in N} E\left(\left|X_{i, j}-E\left(X_{i, j}\right)\right|\right)<\infty$,

2) $\sum_{i, j, i \times j \geq 1}\left(\sum_{k, l, k \times l \leq i \times j} \frac{\operatorname{Cov}\left(X_{i, j}, X_{k, l}\right)}{(i \times j)^{2}}\right)<\infty$.

Then $\frac{S_{m, n}-E\left(S_{m, n}\right)}{m \times n} \stackrel{\text { a.s. }}{\longrightarrow} 0$ as $m \times n \rightarrow \infty$.

Theorem 1.5. (Kim, Baek and Han, [8]) Let $\left(a_{i, j}\right)_{i, j \in N}$ be a 2-dimensional array of positive numbers and $b_{m, n}=\sum_{i=1}^{n} \sum_{j=1}^{m} a_{i, j}$ such that $\frac{a_{m, n}}{b_{m, n}} \rightarrow 0$ and $b_{m, n} \rightarrow \infty$ as $m, n \rightarrow \infty$.

Let $\left(X_{i, j}\right)_{i, j \in N}$ be a 2-dimensional array of pairwise PD random variables with finite variances such that

1) $\sup _{i, j \in N} E\left(\left|X_{i, j}-E\left(X_{i, j}\right)\right|\right)<\infty$,

2) $\sum_{i, j, i \times j \geq 1}\left(\sum_{k, l, k \times l \leq i \times j} \frac{a_{i, j} a_{k, l} \operatorname{Cov}\left(X_{i, j}, X_{k, l}\right)}{b_{i, j}^{2}}\right)<\infty$.

Then $\frac{W_{m, n}-E\left(W_{m, n}\right)}{b_{m, n}} \stackrel{\text { a.s. }}{\longrightarrow} 0$ as $m \times n \rightarrow \infty$ where
$W_{m, n}=\sum_{i=1}^{n} \sum_{j=1}^{m} a_{i, j} X_{i, j}$.

Observe that, for a double indexed sequence of real number $\left(a_{m, n}\right)_{m, n \in N}$, the convergence as $m \times n \rightarrow \infty$ implies the convergence as $m, n \rightarrow \infty$. However, a double sequence $\left(a_{m, n}\right)_{m, n \in N}$ where $a_{m, n}=\frac{(-1)^{m+n}(m+n)}{m \times n}$ shows us that the converse is not true in general.

Our goal is to obtain the SLLN for 2-dimensional array of random variables in case of pairwise ND.

A double sequence $\left(X_{i, j}\right)_{i, j \in N}$ is said to be pairwise negtively dependent (pairwise ND) if for any $a, b \in R$ and $(i, j) \neq(k, l)$,

$$
P\left(X_{i, j}>a, X_{k, l}>b\right) \leq P\left(X_{i, j}>a\right) P\left(X_{k, l}>b\right) .
$$

The followings are SLLNs for a 2-dimensional array of pairwise ND random variables which are all our results.

Theorem 1.6. Let $\left(a_{m}\right)_{m \in N}$ and $\left(b_{n}\right)_{n \in N}$ be increasing sequences of positive numbers such that $a_{m}, b_{n} \geq e$ which $a_{m} \rightarrow \infty$ as $m \rightarrow \infty$ and $b_{n} \rightarrow \infty$ as $n \rightarrow \infty$.

Let $\left(X_{i, j}\right)_{i, j \in N}$ be a 2-dimensional array of pairwise ND random variables with finite variances. If there exist real numbers $p, q$ such that

$$
\sum_{i=1}^{\infty} \sum_{j=1}^{\infty} \frac{\operatorname{Var}\left(X_{i, j}\right)}{a_{i}^{\frac{p}{2}} \times b_{j}^{\frac{q}{2}}}<\infty
$$

then for any double sequence $\left(c_{m, n}\right)_{m, n \in N}$ such that

$$
\begin{aligned}
c_{m, n} \geq a_{m}^{\frac{p}{2}} \times b_{n}^{\frac{q}{2}} \text { for every } m, n \in N, & \\
& \frac{S_{m, n}-E\left(S_{m, n}\right)}{c_{m, n}} \stackrel{\text { a.s. }}{\longrightarrow} 0 \text { as } m \times n \rightarrow \infty .
\end{aligned}
$$

The next theorem is the SLLN for the difference of random variables which independent and identically distributed conditions are regarded.

Theorem 1.7. Let $\left(X_{i, j}\right)_{i, j \in N}$ and $\left(Y_{i, j}\right)_{i, j \in N}$ be 2dimensional arrays of random variables on a probability space $(\Omega, F, P)$. If

$$
\sum_{i=1}^{\infty} \sum_{j=1}^{\infty} P\left\{X_{i, j} \neq Y_{i, j}\right\}<\infty,
$$

then

$$
\frac{1}{m \times n} \sum_{i=1}^{m} \sum_{j=1}^{n}\left(X_{i, j}-Y_{i, j}\right) \stackrel{\text { a.s. }}{\longrightarrow} 0
$$

as $m \times n \rightarrow \infty$. 
Corollary 1.8 and Corollary 1.9 follow directly from Theorem 1.6 by choosing $c_{m, n}=\left(a_{m}+b_{n}\right)^{k}$ and $c_{m, n}=a_{m} \times b_{n}$ where $a_{m}=3 m$ and $b_{n}=3 n$ with $p=q=$ 4 , respectively.

Corollary 1.8. Let $\left(a_{m}\right)_{m \in N}$ and $\left(b_{n}\right)_{n \in N}$ be increasing sequences of positive numbers such that $a_{m}, b_{n} \geq e$ which $a_{m} \rightarrow \infty$ as $m \rightarrow \infty$ and $b_{n} \rightarrow \infty$ as $n \rightarrow \infty$.

Let $\left(X_{i, j}\right)_{i, j \in N}$ be a 2-dimensional array of pairwise ND random variables with finite variances. If there exist $p, q \in N$ such that

$$
\sum_{i=1}^{\infty} \sum_{j=1}^{\infty} \frac{\operatorname{Var}\left(X_{i, j}\right)}{a_{i}^{\frac{p}{2}} \times b_{j}^{\frac{q}{2}}}<\infty,
$$

then for any $k \geq p+q$,

$$
\frac{S_{m, n}-E\left(S_{m, n}\right)}{\left(a_{m}+b_{n}\right)^{k}} \stackrel{\text { a.s. }}{\longrightarrow} 0 \text { as } m \times n \rightarrow \infty .
$$

Corollary 1.9. Let $\left(X_{i, j}\right)_{i, j \in N}$ be a 2-dimensional array of pairwise ND random variables with finite variances. If

$$
\sum_{i=1}^{\infty} \sum_{j=1}^{\infty} \frac{\operatorname{Var}\left(X_{i, j}\right)}{(i \times j)^{2}}<\infty,
$$

then

$$
\frac{S_{m, n}-E\left(S_{m, n}\right)}{81(m \times n)^{2}} \stackrel{\text { a.s. }}{\longrightarrow} 0 \text { as } m \times n \rightarrow \infty \text {. }
$$

\section{Auxiliary Results}

In this section, we present some materials which will be used in obtaining the SLLN's in the next section.

Proposition 2.1. (Móricz, [9]) Let $\left(\lambda_{i, j}\right)_{i, j \in N}$ be a double sequence of positive numbers such that for all $i, j \in N$,

$$
\begin{aligned}
& \lambda_{i+1, j}-\lambda_{i, j} \geq 0, \lambda_{i, j+1}-\lambda_{i, j} \geq 0 \\
& \lambda_{i+1, j+1}-\lambda_{i+1, j}-\lambda_{i, j+1}+\lambda_{i, j} \geq 0,
\end{aligned}
$$

and $\lambda_{i, j} \rightarrow \infty$ as $\max \{i, j\} \rightarrow \infty$.

Let $\left(a_{i, j}\right)_{i, j \in N}$ be a double sequence of real numbers.

Assume that

1) $\sum_{i=1}^{\infty} \sum_{j=1}^{\infty} \frac{a_{i, j}}{\lambda_{i, j}}<\infty$,

2) $\sum_{k=1}^{\infty} \frac{a_{i, k}}{\lambda_{i, k}}<\infty$ for every $i \in N$ and $\sum_{k=1}^{\infty} \frac{a_{k, j}}{\lambda_{k, j}}<\infty$ for every $j \in N$. Then $\frac{1}{\lambda_{m, n}} \sum_{i=1}^{m} \sum_{j=1}^{n} a_{i, j} \rightarrow 0$ as $\max \{m, n\} \rightarrow \infty$.

The following proposition is a Borel-Cantelli lemma for a sequence of double indexed events

Proposition 2.2. Let $\left(E_{i, j}\right)_{i, j \in N}$ be a double sequence of events on a probability space $(\Omega, F, P)$. Then

$$
\sum_{i=1}^{\infty} \sum_{j=1}^{\infty} P\left(E_{i, j}\right)<\infty \Rightarrow P\left\{E_{i, j} \text { i.o. }\right\}=0
$$

where $\left\{E_{i, j}\right.$ i.o. $\}=\bigcap_{k=1}^{\infty}\left(\bigcup_{i, j, i \times j \geq k} E_{i, j}\right)$.

Proof. Let $L \in R$ be such that $L=\sum_{i=1}^{\infty} \sum_{j=1}^{\infty} P\left(E_{i, j}\right)$.

First note that

$$
\sum_{i=1}^{\lfloor\sqrt{k}\rfloor} \sum_{j=1}^{\lfloor\sqrt{k}\rfloor} P\left(E_{i, j}\right) \leq \sum_{i, j, i \times j \leq k} P\left(E_{i, j}\right) \leq \sum_{i=1}^{k} \sum_{j=1}^{k} P\left(E_{i, j}\right)
$$

where $\lfloor\sqrt{k}\rfloor$ denote the greatest integer smaller than or equal $\sqrt{k}$ and hence

$$
\begin{aligned}
L & =\lim _{k \rightarrow \infty} \sum_{i=1}^{\lfloor\sqrt{k}\rfloor\lfloor\sqrt{k}\rfloor} \sum_{j=1} P\left(E_{i, j}\right) \leq \lim _{k \rightarrow \infty} \sum_{i, j, i \times j \leq k} P\left(E_{i, j}\right) \\
& \leq \lim _{k \rightarrow \infty} \sum_{i=1}^{k} \sum_{j=1}^{k} P\left(E_{i, j}\right)=L .
\end{aligned}
$$

Therefore $\lim _{k \rightarrow \infty} \sum_{i, j, i \times j \leq k} P\left(E_{i, j}\right)=L$ and

$$
\begin{aligned}
P\left\{E_{i, j} \text { i.o. }\right\} & =\lim _{k \rightarrow \infty} P\left(\bigcup_{i, j, i \times j \geq k} E_{i, j}\right) \leq \lim _{k \rightarrow \infty} \sum_{i, j, i \times j \geq k} P\left(E_{i, j}\right) \\
& =\lim _{k \rightarrow \infty}\left(\sum_{i=1}^{\infty} \sum_{j=1}^{\infty} P\left(E_{i, j}\right) \sum_{i, j, i \times j \leq k-1} P\left(E_{i, j}\right)\right)=0 .
\end{aligned}
$$

This completes the proof. $\square$

\section{Proof of Main Results}

\section{Proof of Theorem 1.6}

Let $m, n \in N$ and define $f(m)=\left\lfloor\ln a_{m}\right\rfloor$ and $g(n)=\left\lfloor\ln b_{n}\right\rfloor$.

Clearly, $f$ and $g$ are increasing whose facts

$f(m) \leq \ln a_{m}<f(m)+1$ and $g(n) \leq \ln b_{n}<g(n)+1$ which imply that $e^{f(m)} \leq a_{m}<e^{f(m)+1}$ and $e^{g(n)} \leq b_{n}<e^{g(n)+1}$.

Let $\varepsilon>0$ be given. By using the fact that $\operatorname{Cov}\left(X_{i, j}, X_{k, l}\right) \leq 0$ for $(i, j) \neq(k, l) \quad([10]$, p. 313), we have

$$
\operatorname{Var}\left(\sum_{i=1}^{m} \sum_{j=1}^{n} X_{i, j}\right) \leq \sum_{i=1}^{m} \sum_{j=1}^{n} \operatorname{Var}\left(X_{i, j}\right) .
$$

From this fact and Chebyshev's inequality, we have 


$$
\begin{aligned}
& \sum_{m=1}^{\infty} \sum_{n=1}^{\infty} P\left\{\frac{\left|S_{m, n}-E\left(S_{m, n}\right)\right|}{C_{m, n}} \geq \varepsilon\right\} \\
& \leq C \sum_{m=1}^{\infty} \sum_{n=1}^{\infty} \frac{\operatorname{Var}\left(S_{m, n}\right)}{C_{m, n}^{2}} \leq C \sum_{m=1}^{\infty} \sum_{n=1}^{\infty} \frac{1}{C_{m, n}^{2}} \sum_{i=1}^{m} \sum_{j=1}^{n} \operatorname{Var}\left(X_{i, j}\right) \\
& \leq C \sum_{i=1}^{\infty} \sum_{j=1}^{\infty} \operatorname{Var}\left(X_{i, j}\right) \sum_{m=i}^{\infty} \sum_{n=j}^{\infty} \frac{1}{C_{m, n}^{2}} \\
& \leq C \sum_{i=1}^{\infty} \sum_{j=1}^{\infty} \operatorname{Var}\left(X_{i, j}\right) \sum_{m=i}^{\infty} \sum_{n=j}^{\infty} \frac{1}{a_{m}^{p} \times b_{n}^{q}} \\
& \leq C \sum_{i=1}^{\infty} \sum_{j=1}^{\infty} \operatorname{Var}\left(X_{i, j}\right) \sum_{m=i}^{\infty} \sum_{n=j}^{\infty} \frac{1}{e^{(p f(m)+q g(n))}}
\end{aligned}
$$

For each $i, j \in N$, let

$$
\begin{aligned}
& A_{i}=\left\{s \in N: e^{f(s)+1} \geq a_{i}\right\} \\
& B_{j}=\left\{t \in N: e^{f(t)+1} \geq b_{j}\right\}
\end{aligned}
$$

and $\tilde{i}=\min A_{i}$ and $\tilde{j}=\min B_{j}$. Since $i \in A_{i} \quad$ and $j \in B_{j}$, we have $\tilde{i} \leq i$ and $\tilde{j} \leq j$. From this facts and (3.1), we have

$$
\begin{aligned}
& \sum_{m=1}^{\infty} \sum_{n=1}^{\infty} P\left\{\frac{\left|S_{m, n}-E\left(S_{m, n}\right)\right|}{C_{m, n}} \geq \varepsilon\right\} \\
& \leq C \sum_{i=1}^{\infty} \sum_{j=1}^{\infty} \operatorname{Var}\left(X_{i, j}\right) \sum_{m=\tilde{i}}^{\infty} \sum_{n=\tilde{j}}^{\infty} \frac{1}{e^{(p f(m)+q g(n))}} \\
& \leq C \sum_{i=1}^{\infty} \sum_{j=1}^{\infty} \operatorname{Var}\left(X_{i, j}\right) \sum_{m=f(\tilde{i})}^{\infty} \sum_{n=g(\tilde{j})}^{\infty} \frac{1}{e^{\left.\frac{p m+q n}{2}\right)}} \\
& \leq C \sum_{i=1}^{\infty} \sum_{j=1}^{\infty} \operatorname{Var}\left(X_{i, j}\right) \sum_{m=f(\tilde{i})}^{\sum^{\frac{p m}{2}}} \frac{1}{\sum_{n=g(\tilde{j})}^{\infty} \frac{1}{e^{\frac{q n}{2}}}} \\
& \leq C \sum_{i=1}^{\infty} \sum_{j=1}^{\infty} \operatorname{Var}\left(X_{i, j}\right)\left(\frac{1}{e^{\frac{p f(\tilde{i})}{2}}}\right)\left(\frac{1}{e^{\frac{q g(\tilde{j})}{2}}}\right) .
\end{aligned}
$$

Since $\tilde{i} \in A_{i}$ and $\tilde{j} \in B_{j}$, we have $\frac{1}{e^{f(\tilde{i})}}<e \cdot \frac{1}{a_{i}}$ and $\frac{1}{e^{g(\tilde{j})}}<e \cdot \frac{1}{b_{j}}$.

From this facts and (3.2) together with our assumption 2), we have

$$
\sum_{m=1}^{\infty} \sum_{n=1}^{\infty} P\left\{\frac{\left|S_{m, n}-E\left(S_{m, n}\right)\right|}{C_{m, n}} \geq \varepsilon\right\} \leq C \sum_{i=1}^{\infty} \sum_{j=1}^{\infty} \frac{\operatorname{Var}\left(X_{i, j}\right)}{a_{i}^{\frac{p}{2}} \times b_{j}^{\frac{q}{2}}}<\infty .
$$

By Proposition 2.2 with

$$
E_{m, n}=\left\{\frac{\left|S_{m, n}-E\left(S_{m, n}\right)\right|}{c_{m, n}} \geq \varepsilon\right\},
$$

we have $P\left\{E_{m, n}\right.$ i.o. $\}=0$ and this hold for every $\varepsilon>0$. By using the same idea with Theorem 4.2.2 ([11], p. 77), we can prove that

$$
\frac{S_{m, n}-E\left(S_{m, n}\right)}{C_{m, n}} \stackrel{\text { a.s. }}{\longrightarrow} 0 \text { as } m \times n \rightarrow \infty .
$$

\section{Proof of Theorem 1.7}

Let $\Omega_{0}=\bigcap_{k=1}^{\infty} \bigcup_{i, j, i \times j \geq k}\left\{X_{i, j} \neq Y_{i, j}\right\}$. By Proposition 2.2, we have

$$
P\left(\Omega_{0}^{c}\right)=1-P\left(\Omega_{0}\right)=1-P\left(\left\{X_{i, j} \neq Y_{i, j}\right\} \text { i.o. }\right)=1 .
$$

For every $\omega \in \Omega_{0}^{c}$, we will show that

$$
\sum_{i=1}^{\infty} \sum_{j=1}^{\infty} \frac{1}{i \times j}\left(X_{i, j}(\omega)-Y_{i, j}(\omega)\right)<\infty,
$$

for every $i \in N$,

$$
\sum_{j=1}^{\infty} \frac{1}{i \times j}\left(X_{i, j}(\omega)-Y_{i, j}(\omega)\right)<\infty
$$

and for every $j \in N$,

$$
\sum_{i=1}^{\infty} \frac{1}{i \times j}\left(X_{i, j}(\omega)-Y_{i, j}(\omega)\right)<\infty .
$$

From (3.3), (3.4) and (3.5), we can apply Proposition 2.1 with $\lambda_{i, j}=i \times j$ that

$$
\frac{1}{m \times n} \sum_{i=1}^{m} \sum_{j=1}^{n}\left(X_{i, j}-Y_{i, j}\right) \stackrel{\text { a.s. }}{\longrightarrow} 0
$$

as $\max \{m, n\} \rightarrow \infty$. We here note that $a_{m, n} \rightarrow a$ as $\max \{m, n\} \rightarrow \infty$ implies $a_{m, n} \rightarrow a$ as $m \times n \rightarrow \infty$. Hence

$$
\frac{1}{m \times n} \sum_{i=1}^{m} \sum_{j=1}^{n}\left(X_{i, j}-Y_{i, j}\right) \stackrel{\text { a.s. }}{\longrightarrow} 0
$$

as $m \times n \rightarrow \infty$.

To prove (3.3), (3.4) and (3.5), let $\omega \in \Omega_{0}^{c}$. Then there exists $k_{\omega} \in N$ such that for $i, j \in N$,

$$
i \times j \geq k_{\omega} \Rightarrow X_{i, j}(\omega)=Y_{i, j}(\omega) \text {. }
$$

Thus for each $\omega \in \Omega_{0}^{c},\left(X_{i, j}(\omega)\right)_{i, j \in N}$ and $\left(Y_{i, j}(\omega)\right)_{i, j \in N}$ are different only finitely many terms. This implies that (3.3) holds.

For fixed $i \in N$, we can find a large $j_{0} \in N$ such that (3.6) holds for all $j \geq j_{0}$ which means that there are only finitely many different terms of $\left(X_{i, j}(\omega)\right)_{i, j \in N}$ and $\left(Y_{i, j}(\omega)\right)_{i, j \in N}$. So for fixed $i \in N$,

$$
\sum_{j=1}^{\infty} \frac{1}{i \times j}\left(X_{i, j}(\omega)-Y_{i, j}(\omega)\right)<\infty .
$$


Similarly, for fixed $j \in N$,

$$
\sum_{i=1}^{\infty} \frac{1}{i \times j}\left(X_{i, j}(\omega)-Y_{i, j}(\omega)\right)<\infty .
$$

Now (3.4) and (3.5) are now proved and this ends the proof. $\square$

Remark 3.1. In case of $m$ fixed and $n \rightarrow \infty$, by considering the limit as $m \times n \rightarrow \infty$, we also obtain the corresponding results for a case of 1-dimensional pairwise ND random variables.

\section{Example}

Example 4.1 A box contains $p q$ balls of $p$ different colors and $q$ different sizes in each color. Pick 2 balls randomly.

Let $\tilde{X}_{i, j}, i=1,2, \cdots, p$ and $j=1,2, \cdots, q$ be a random variable indicating the presence of a ball of the $i^{\text {th }}$ color and the $j^{\text {th }}$ size such that

$\tilde{X}_{i, j}=\left\{\begin{array}{l}1, \text { if the } i^{\text {th }} \text { color and the } j^{\text {th }} \text { size of ball is picked, } \\ 0, \text { otherwise. }\end{array}\right.$ by

For $i, j \in N$, let $X_{i, j}$ be a random variable defined

$$
X_{i, j}=\left\{\begin{array}{l}
\tilde{X}_{i, j}, \text { if } 1 \leq i \leq p \text { and } 1 \leq j \leq q, \\
0, \quad \text { otherwise. }
\end{array}\right.
$$

Proof. By a direct calculation, we have $X_{i, j}$ 's are pairwise ND random variables, i.e. for $i, j, k, l \in R$ that $(i, j) \neq(k, l)$ and $a, b \in R$,

$$
P\left(X_{i, j}>a, X_{k, l}>b\right) \leq P\left(X_{i, j}>a\right) P\left(X_{k, l}>b\right) .
$$

Note that

$$
E\left(X_{i, j}\right)=\frac{p q-1}{\left(\begin{array}{c}
p q \\
2
\end{array}\right)}=\frac{2}{p q}
$$

and $\operatorname{Var}\left(X_{i, j}\right)=\frac{2}{p q}-\frac{4}{(p q)^{2}}$.

Hence,

$$
\begin{aligned}
\sum_{i=1}^{\infty} \sum_{j=1}^{\infty} \frac{\operatorname{Var}\left(X_{i, j}\right)}{(i \times j)^{2}} & =\lim _{m, n \rightarrow \infty}\left(\sum_{i=1}^{m} \sum_{j=1}^{n} \frac{\operatorname{Var}\left(X_{i, j}\right)}{(i \times j)^{2}}\right) \\
& =\left(\frac{2}{p q}-\frac{4}{(p q)^{2}}\right) \sum_{i=1}^{\infty} \sum_{j=1}^{\infty} \frac{1}{(i \times j)^{2}}<\infty .
\end{aligned}
$$

By applying Theorem 1.6, for any double sequence $\left(c_{m, n}\right)_{m, n \in N}$ such that $c_{m, n} \geq 81(m \times n)^{2}$ for every $m$ $n \in N$, we have $\frac{S_{m, n}-E\left(S_{m, n}\right)}{C_{m, n}} \stackrel{\text { a.s. }}{\longrightarrow} 0$ as $m \times n \rightarrow \infty$.

\section{Acknowledgements}

The authors would like to thank referees for valuable comments and suggestions which have helped improving our work. The first author gives an appreciation and thanks to the Institute for the Promotion of Teaching Science and Technology for financial support.

\section{REFERENCES}

[1] S. Csörgő, K. Tandori and V. Totik, "On the Strong Law of Large Numbers for Pairwise Independent Random Variables," Acta Mathematica Hungarica, Vol. 42, No. 34, 1983, pp. 319-330. doi:10.1007/BF01956779

[2] N. Etemadi, "An Elementary Proof of the Strong Law of Large Numbers," Zeitschrift für Wahrscheinlichkeitstheorie und Verwandte Gebiete, Vol. 55, No. 1, 1981, pp. 119-122.

[3] R. G. Laha and V. K. Rohatgi, "Probability Theory," John Wiley \& Sons, Hoboken, 1979.

[4] T. Birkel, "A Note on the Strong Law of Large Numbers for Positively Dependent Random Variables," Statistics \& Probability Letters, Vol. 7, No. 1, 1989, pp. 17-20. doi:10.1016/0167-7152(88)90080-6

[5] H. A. Azarnoosh, "On the Law of Large Numbers for Negatively Dependent Random Variables," Pakistan Journal of Statistics, Vol. 19, No. 1, 2003, pp. 15-23.

[6] H. R. Nili Sani, H. A. Azarnoosh and A. Bozorgnia, "The Strong Law of Large Numbers for Pairwise Negatively Dependent Random Variables," Iranian Journal of Science \& Technology, Vol. 28, No. A2, 2004, pp. 211-217.

[7] T. S. Kim, H. Y. Beak and H. Y. Seo, "On Strong Laws of Large Numbers for 2-Dimensional Positively Dependent Random Variables," Bulletin of the Korean Mathematical Society, Vol. 35, No. 4, 1998, pp. 709-718.

[8] T. S. Kim, H. Y. Beak and K. H. Han, "On the Almost Sure Convergence of Weighted Sums of 2-Dimensional Arrays of Positive Dependent Random Variables," Communications of the Korean Mathematical Society, Vol. 14, No. 4, 1999, pp. 797-804.

[9] F. Móricz, "The Kronecker Lemmas for Multiple Series and Some Applications," Acta Mathematica Academiae Scientiarum Hungaricae, Vol. 37, No. 1-3, 1981, pp. 3950. doi:10.1007/BF01904871

[10] N. Ebrahimi and M. Ghosh, "Multivariate Negative Dependence," Communications in Statistics-Theory and Methods, Vol. A10, No. 4, 1981, pp. 307-337.

[11] K. L. Chung, "A Course in Probability Theory," Academic Press, London, 2001. 